\title{
The inversion of the Laplace transformation by a direct expansion in series and its application to boundary-value problems.
}

By

R. V. Churchill (University of Michigan) at Freiburg i. B.

$\S 1$.

Introduction.

The one-sided Laplace transformation of a function $F(t)$,

$$
\mathfrak{L}\{F(t)\}=\int_{0}^{\infty} e^{-s t} F(t) d t=f(s),
$$

transforms the operation of differentiation of $F(t)$ into an algebraic operation on $f(s)$ in the following way:

(2) $\mathfrak{L}\left\{F^{(n)}(l)\right\}=s^{n} f(s)-s^{n-1} F^{\prime}(0)-s^{n-2} F^{\prime}(0)-\ldots-F^{(n-1)}(0)$,

where the indices in parentheses denote derivatives ${ }^{1}$ ). This gave rise to an important method (used by Doetsch ${ }^{2}$ ) since 1923) of solving linear boundary-value problems in partial differential equations; for if the transformation can be applied to the unknown function with respect to one of the independent variables, the problem in the transformed function does not involve derivatives with respect to that variable. A twodimensional problem is thus transformed ${ }^{3}$ ) into one in ordinary diffe-

1) For conditions under which this property of the transformation is valid sec G. Doetsch, Die Integrodifferentialgleichungen vom Faltungstypus. Math. Annalen 89 (1923), 192-207. [p. 198, Theorem III]. Here the Laplace transformation of $F^{(n)}(t)$ is assumed absolutely convergent; only simple convergence is used in Doetsch's more recent derivation of this property: Der Faltungssatz in der Theorie der Laplace-Transformation. Annali della R. Scuola Norm. Sup. di Pisa (2) 4 (1935), $71-84$ [Lemma 2].

2) See for instance the series of papers: G. Doetsch, Probleme aus der Theorie der Wärmeleitung. Math. Zeitschr. 22 (1925), 285-292; 293-306; 25 (1926)s $608-626 ; 96(1927), 89-98 ; 28(1928), 567-578$. In the first and fourth papers of this series F. Bernstein is a co-author. Also see other references in G. Doetsch, Les équations aux dérivées partielles du type parabolique. L'Enseignement Mathé. matique 35 (1936), $43-87$ [p. 86, bibliography].

$\left.{ }^{3}\right)$ discussion of the hypotheses on which such transformations of boundaryvalue problems depend is given in the last paper under ${ }^{2}$ ); also in G. Doetseh, Elektrische Schwingungen in einem anfänglich strom- und spannungslosen Kabel anter dem EinfluB einer Randerregung. - Festschrift d. Techn. Hoehschule Stuttgart zur Vollendung ihres ersten Jahrhunderts. Verlag Springer, 1929, pp. 56-78. 
rential equations involving a parameter $s$. The solution of the original problem is obtained by applying the inverse of the Laplace transformation to the solution of the transformed problem. This is usually the most difficult step in the process; it is this step which concerns us in this paper.

The inversion process with which we are principally concerned here is one which expresses the inverse $\mathfrak{L}^{-1}\{f(s)\}$ of the Laplace transformation in a series ${ }^{4}$ ). Representations of this general character have usually been written, especially in electrical engineering, by the formal process of expanding $f(s)$ in some series and applying the operator $\mathbb{8}^{-1}$ termwise, but this method leads to incorrect results in many cases when the series is infinite ${ }^{5}$ ).

The particular series which is shown here to represent $\mathfrak{L}^{-1}\{f\}$, for functions $f(s)$ of a certain class, is a somewhat generalized form of the Heaviside expansion formula ${ }^{6}$ ). It is important to have usable sets of conditions on $f(s)$ under which this series expansion is valid, for here too it frequently happens that a formal application of this series fails to give the inverse transformation.

In order to establish the direct expansion of $\mathcal{L}^{-1}\{t\}$ in series we employ here a well-known expression for this transformation as an integral in the complex plane of the variables. It was found possible to improve upon the already known conditions on $f(s)$ which are sufficient for this integral representation. These new conditions are established in $\S 2$. They are used in $\$ 3$ to establish the series representation under different sets of conditions. The two sets of conditions which are most useful in solving boundary-value problems are given in Theorems 4 and 5. In $\S 4$ the inverse transformations of two particular functions are found with the help of the expansion in series, and these are used in $\$ 5$ to find a simple solution of a problem in the forced displacements in a bar.

4) Concerning asymptotic representations of $\mathfrak{I}^{-1}\{f\}$ for large values of $t$ see G. Doetsch, Ein allgemeines Prinzip der asymptotisehen Intwicklung, Journ. f. d. reine u. angewandte Matb. 167 (1932), 274-293 [p. 286 ff.]. This paper also contains some interesting observations on the inversion of the Laplace transformation by means of the integral in the complex plane [pp. 278, 279].

5) Such a process, together with a method of verifying the results, is illustrated by R. V. Churchill, Temperature distribution in a slab of two layers. Duke Math. Journ. 2 (1936), 405-4.14.

6) Relations between the methods of operational calculus and functional transformations are digeaseed in G. Doetsch, Die Anwendung von Funktionaltransformationen in der Theerie der Differentialgleichungen und die symbolische Methode (Operatorenkalkü), Jahresber. D. M. V. 48 (1934), 238-253, and in other papers cited there. 


\section{$\S 2$.}

Sufficient conditions for representing $\mathfrak{g}^{-1}\{f\rangle$ by an integral.

The inverse Laplace transformation of a function $f(s)$,

$$
\mathfrak{2}^{-1}\{f(s)\}=F(t)
$$

is a function $\left.F(t)^{7}\right)$ which satisties the integral equation

$$
f(s)=\int_{0}^{\infty} e^{-s t} F(t) d t .
$$

Conditions under which $f(s)$ is representable in the form (3), together with an expression for $F(t)$ in terms of $f(s)$, where published in 1920 by M. Fujiwara ${ }^{8}$ ). But his proof assumes that $f(s)$ satisfies some conditions not stated in his theorems. Instead of following Fujiwara's method of proof throughout, after stating the complete set of conditions used, it is simpler to give the theorem and proof in the following form, proposed to the author by G. Doetsch:

Theorem 1. Let $f(s)$ satisfy the following conditions:

$1^{\circ} f(s)$ is analytic in the half-plane $\Re(s)>\alpha$, where $\alpha$ is a real constant. $2^{\circ}$ For a fixed $y>\alpha$

$$
\lim _{\omega \rightarrow \infty} \int_{\gamma-\omega i}^{\gamma+\omega i} e^{t z} f(z) d z
$$

converges uniformly in each finite interval $0 \leqq t \leqq T$.

$3^{0} \lim _{\omega \rightarrow \infty} \int_{\gamma-\omega i}^{\gamma+\omega i} \frac{|f(z)|}{1+|z|}|d z|$ converges.

$4^{0}|f(s)|$ is bounded in $\mathfrak{R}(s) \geqq \gamma$.

$5^{0} \lim _{i \rightarrow \infty}|f(\sigma+\omega i)|=0$ uniformly for all $\omega$.

Then for all $s$ with $\Re(s)>\gamma$ it follows that

where

$$
f(s)=\int_{0}^{\infty} e^{-8 t} F(t) d t
$$

$$
F(t)=\frac{1}{2 \pi i} \lim _{w \rightarrow \infty} \int_{\gamma-\omega i}^{\gamma+\omega i} e^{\xi z} f(z) d z .
$$

7) Concerning the uniqueness of $F(t)$ see Lerch, Sur un point de la théorie des fonctions génératrices d'Abel, Acta Math. 27 (1903), 339-351.

8) M. Fujiwara, Ủber Abelsche erzeugende Funktion und Darstellbarkeitsbedingung von Funktionen durch Dirichletsche Reihen, The Tôhoku Math. Journ. 17 (1920), 363-383, [Theorems I and II]. 
As a result of the uniform convergence assumed in $2^{0}$ it follows that

$$
\begin{aligned}
\int_{0}^{T} e^{-s t} F(t) d t & =\int_{0}^{T} e^{-s t} d t\left\{\frac{1}{2 \pi i} \lim _{\omega \rightarrow \infty} \int_{\gamma-\omega i}^{\gamma+\omega i} e^{t z} f(z) d z\right\} \\
& \left.=\frac{1}{2 \pi i} \lim _{\omega \rightarrow \infty} \int_{\gamma-\omega i}^{\gamma+\omega i} \frac{1-e^{-(8-z) r}}{8-z} f(z) d z^{9}\right) .
\end{aligned}
$$

According to the hypothesis $3^{0}$ this limit converges uniformly in $T$ for all $T>0$, for

$$
\lim _{\omega \rightarrow \infty} \int_{\gamma-\omega i}^{\gamma+\omega i}\left|\frac{1-e^{-(z-z) T}}{s-z}\right||f(z) d z| \leqq \lim _{\omega \rightarrow \infty} \int_{\gamma=\omega i}^{\gamma+\omega i} \frac{2|f(z)|}{|z-s|}|d z|,
$$

and for each $s$ a sufficiently large $|\omega|$ can be found, say $\Omega$, such that for $|\omega| \geqq \Omega$ this integrand is less than $\frac{4|f(z)|}{|z|+1}$. The limit as $T \rightarrow \infty$ can thus be taken under the integral sign:

$$
\int_{0}^{\infty} e^{-s t} F(t) d t=\frac{1}{2 \pi i} \lim _{\omega \rightarrow \infty} \int_{\gamma-\omega i}^{\gamma+\omega i} \frac{f(z)}{s-z} d z .
$$

To show that this last limit is $f(s)$ under the hypotheses stated apply the theorem of Cauchy, integrating $\frac{f(z)}{s-z}$ around the rectangle with vertices $\gamma+\omega i, \gamma-\omega i, \omega-\omega i, \omega+\omega i$. Let a fixed $\Omega$ be chosen so large that $|f(s)|<\varepsilon$ to the right of the line $\Re(z)=\Omega$; this is possible according to $5^{0}$. Then for the integral along the right-hand side of the rectangle it follows that, for $\omega>\Omega$,

$$
\left|\int_{\omega,-\infty i}^{\omega+\omega i} \frac{f(z)}{s-z} d z\right|<\varepsilon \frac{2(\omega)}{\omega-\mathbb{R}(s)} .
$$

Along the segment of the upper side to the right of the line $\mathfrak{R}(z)=\Omega$,

$$
\left|\int_{\omega+\omega i}^{\Omega+\omega i} \frac{f(z)}{s-z} d z\right| \leqq \varepsilon \frac{\omega-\Omega}{\omega-\mathfrak{J}(s)}
$$

9) The Cauchy principal value can be written as an infinite integral:

$$
\lim _{\omega \rightarrow \infty} \int_{\gamma-\omega i}^{\gamma+\omega i} e^{i z} f(z) d z=i \int_{0}^{\infty}\left[e^{t(\gamma+\omega i)} f(\gamma+\omega i)+e^{t(\gamma-\omega t)} f(\gamma-\omega i)\right] d \omega .
$$


and along the remaining portion of the upper side,

$$
\left|\int_{\Omega+\omega i}^{\gamma+\omega i} \frac{f(z)}{s-z} d z\right| \leqq M \frac{\Omega-\gamma}{\omega-\mathfrak{J}(s)}
$$

where $M$ is an upper bound of $|f(s)|$ in $R(s) \geqq \gamma$ (condition $4^{0}$ ); similarly for the integration over the two portions of the lower side. Hence by first selecting $\Omega$ sufficiently large and then taking $\omega$ large enough the integrals over these three sides of the rectangle can be made less than any fixed $\varepsilon^{\prime}>0$. Since

$$
\frac{1}{2 \pi i} \oint \frac{f(z)}{z-s} d z=f(s)
$$

when the integral is taken around any rectangle with $s$ in its interior, it follows that

$$
\frac{1}{2 \pi i} \lim _{\omega \rightarrow \infty} \int_{\gamma-\omega i}^{\gamma+\omega i} \frac{f(z)}{s-z} d(z)=f(s)
$$

and the theorem is proved.

It should be observed that a necessary condition in order that $f(s)$ shall have a real inverse transformation $F(t)$ is that

$$
f(\bar{s})=\overline{f(s)}
$$

where the bar denotes the conjugate complex number. This follows immediately from the representation (3) of $f(s)$ as an integral. Hence if $F(t)$ is real, as is the case in the applications to boundary-value problems, we can write

$$
\lim _{\omega \rightarrow \infty} \int_{\gamma-\omega i}^{\gamma+\omega i} e^{z t} f(s) d s=2 i \int_{0}^{\infty} \Re\left[e^{t(\gamma+\omega i)} f(\gamma+\omega i)\right] d \omega_{.}
$$

The three conditions $3^{0}, 4^{0}, 5^{0}$ in Theorem 1 can be replaced by the single condition that for some fixed $k>0,\left|s^{k} f(s)\right|$ is bounded in $\mathfrak{R}(s)$ $\geqq \gamma$. This is simpler to use, but more restrictive.

The following is an important special case of Theorem 1.

Theorem 2. Let $f(s)$ be analytic in the half-plane $\mathfrak{R}(s)>\alpha$, and for a fixed $y>\alpha$ let

$$
\int_{-\infty}^{\infty}|f(y+\omega i)| d \omega
$$

be convergent. Also let $|f(s)|$ be bounded in $\Re(s) \geqq \gamma$ and $\lim _{\sigma \rightarrow \infty}|f(\sigma+\omega i)|=0$ uniformly for all $\omega$. Then for each $s$ such that $\Re(s)>\gamma$,

$$
f(s)=\int_{0}^{\infty} e^{-s t} F(t) d t
$$


where

$$
F(t)=\frac{1}{2 \pi i} \int_{\gamma-\infty i}^{\gamma+\infty i} e^{t z} f(z) d z
$$

The assumption of absolute convergence here insures the uniform convergence of the integral in condition $2^{0}$, Theorem 1 , for when $z=\gamma+\omega i$,

$$
\left|e^{t z} f(z)\right| \leqq e^{T|\gamma|}|f(y+\omega i)| \text {. }
$$

The other conditions in Theorem 1 are clearly satisfied.

The conditions here are much simpler than those in Theorem 1. But the condition of absolute convergence of the infinite integral here is much narrower than the condition $2^{0}$ of Theorem 1 , that the Cauchy principal value of the integral converge uniformly.

Sufficient conditions for the inversion in the form of a complex integral have also been established by Tamarkin ${ }^{10}$ ). His second set of conditions form a special case of those in Theorem 2; he assumes that $\alpha \geqq 0$ and that $|f(s)|$ approaches zero uniformly in $\Re(s) \geqq \gamma$ as $|s|$ becomes infinite ${ }^{11}$ ).

\section{$\S 3$.}

\section{Sufficient conditions for representing $8^{-1}\{f\}$ by a series.}

A direct expansion of the inverse Laplace transformation in series will now be established from the integral form (4). The series is more appropriate in many problems than the integral.

Theorem 3. Let $f(s)$ be analytic in the half-plane $\Re(s) \geqq \gamma$ and admit (on the line $\Re(s)=\gamma$ ). the integral form (4) of its inverse Laplace transformation; also let its singular points consist only of poles $s_{m}$ of order $\sigma_{m}$. If there is a fixed number $k>0$ for which $\left|s^{k} f(s)\right|$ is bounded at all points (in $R(s) \leqq \gamma$ ) on some sequence of circles $|s|=\varrho_{n}$, where $\varrho_{n}$ becomes infinite with $n$, then

$$
\mathfrak{L}^{-1}\{f(s)\}=\sum_{m=1}^{\infty} e^{\varepsilon_{m} t} \sum_{p=1}^{\sigma_{m}} A_{m p} t^{p-1} /(p-1) ! \quad \text { for } t>0
$$

10) J. D. Tamarkin, On Laplace's integral equations, Trans. Am. Math. Soc. 28 (1926), 417-425. One of the conditions in his first set is that the order of integration can be interchanged in the iterated infinite integral.

11) The class of analytic functions $f(s)$ which can be represented as Laplace transforms $f(s)=\mathfrak{L}\{F\}$ with $F$ expressed as a complex integral can be exactly determined by function-theoretic conditions, if one assumes $F$ to be integrable in the $p^{\text {th }}$ power $(p>1)$ in the Lebesgue sense and if one replaces simple convergence of the complex integral by convergence in the mean, as is shown in a recent paper by G. Doetsch, Bedingungen für die Darstellbarkeit einer Funktion als Laplace-Integral und eine Umkehrformel für die Laplace-Transformation, Math. Zoitschr. 42 (1937). 
The numbers $A_{m p}$ are coefficients in the Laurent expansion of $f(s)$ about the pole $s_{m}$ :

$$
f(s)=\sum_{p=1}^{\sigma_{m}} A_{m p} /\left(s-s_{m}\right)^{p}+\sum_{n=0}^{\infty} B_{m n}\left(s-s_{m}\right)^{n}
$$

and the terms in the series (5) which correspond to poles lying between two consecutive circles $|s|=\varrho_{n-1}$ and $|s|=\varrho_{n}$ are grouped as a single term.

When the terms of the series (6) are multiplied by $e^{s t}$ it is readily seen that the residue of $e^{s t} f(s)$ at the pole $s_{m}$ is

$$
e^{s_{m} \ell} \sum_{p=1}^{\sigma_{m}} A_{m p} t^{p-1} /(p-1) !
$$

Put $s=\varrho e^{i t}$ and let $C_{n}$ denote that arc of the circle $\varrho=\varrho_{n}$ which lies to the left of the line $\Re(s)=\gamma$; also let $P_{n}$ denote the segment of this line intercepted by the circle. Then according to the Caruchy theorem of resifues

$$
\begin{aligned}
\frac{1}{2 \pi i} \int_{\left(\mathcal{P}_{n}\right)} e^{s t} f(s) d s & +\frac{1}{2 \pi i} \int_{\left(\hat{O}_{n}\right)} e^{s t} f(s) d s \\
= & \sum_{m=1}^{m_{n}} e^{s m} \sum_{p=1}^{\sigma_{m}} A_{m p} t^{p-1} /(p-1) !
\end{aligned}
$$

where $m_{n}$ is the number of poles within $\varrho=\varrho_{n}$, and the sense of the integration is positive.

The second integral in (7) approaches zero as $n$ becomes infinite, for $t>0$. For if $M$ is an upper bound of $\left|s^{k} f(s)\right|$ corresponding to a fixed $k>0$ for all points on the arcs $C_{n}$, then

$$
\left|\int_{\left(C_{n}\right)} f(s) e^{s t} d s\right| \leqq \varrho_{n} \int_{\frac{\pi}{2}-\theta_{n}}^{\frac{3 \pi}{2}+\theta_{n}}\left|f\left(\varrho_{n} e^{i \theta}\right)\right| e^{t \rho_{n} \cos \theta} d \theta \leqq \varrho_{n}^{1-k} M \int_{\frac{\pi}{2}-\theta_{n}}^{\frac{3 \pi}{2}+\theta_{n}} e^{i \ell_{n} \cos \theta} d \theta
$$

where

$$
\theta_{n}=\arcsin \frac{\gamma}{\varrho_{n}} \quad\left(-\frac{\pi}{2}<\theta_{n}<\frac{\pi}{2}\right) .
$$

The last member of this inequality can be written

$$
J=2 \varrho_{n}^{1-k} M \int_{\frac{\pi}{2}-\theta_{n}}^{\pi} e^{t \varrho_{n} \cos \theta} d \theta=2 \varrho_{n}^{1-k} M \int_{-\theta_{n}}^{\pi / 2} e^{-t \varrho_{n} \sin \theta} d \theta .
$$


Suppose first that $\gamma \leqq 0$. Since $\sin \theta>\theta / 2$ when $0<\theta<\pi / 2$, then

$$
\tilde{J}<2 \varrho_{n}^{1-k} M \int_{-\theta_{n}}^{\pi / 2} e^{-t \rho_{n} \theta / 9} d \theta=\frac{4 M}{t \varrho_{n}^{k}}\left(e^{t \rho_{n} \theta_{n} / 9}-e^{-t \varrho_{n} \pi / 4}\right) .
$$

But $\varrho_{n} \theta_{n} \rightarrow \gamma$ as $n \rightarrow \infty$ and hence $J \rightarrow 0$ as $n \rightarrow \infty,(\gamma \leqq 0)$. When $\gamma>0$ the integral $J$ can be written

$$
J=2 \varrho_{n}^{1-k} M\left(\int_{0}^{\pi / 9} e^{-t \varrho_{n} \sin \theta} d \theta+\int_{0}^{\theta_{n}} e^{t \varrho_{n} \sin \theta} d \theta\right)
$$

and since $\theta / 2<\sin \theta<\theta$ when $0<\theta<\pi / 2$,

$$
\begin{aligned}
J<2 \varrho_{n}^{1-k} M & \left(\int_{0}^{\pi / 2} e^{-t \varrho_{n} \theta / 2} d \theta+\int_{0}^{\theta_{n}} e^{t \varrho_{n} \theta} d \theta\right) \\
& =2 M\left(1-2 e^{-t \varrho_{n} \pi / 4}+e^{t \varrho_{n} \theta_{n}}\right) /\left(t \varrho_{n}^{k}\right) .
\end{aligned}
$$

Hence $J \rightarrow 0$ as $n \rightarrow \infty,(\gamma>0)$, so

$$
\lim _{n \rightarrow \infty} \int_{\left(C_{n}\right)} e^{s t} f(s) d s=0 \quad \text { for } t>0 .
$$

Since the first integral in (7) converges to $g^{-1}\{f(s)\}$ as $n$ becomes infinite according to the conditions in the theorem, the sum on the right also converges to this value and the theorem is proved.

The sum in the right-hand member of (7) contains just those terms which correspond to the poles of $f(s)$ lying inside the circle $\varrho=\varrho_{n}$, so if the resulting series is infinite it converges to $\mathfrak{R}^{-1}\{f\}$ when the terms are grouped according to these circles.

To show then that the inverse Laplace transformation of a given function $f(s)$ is represented by the series (5) for this function, it is sufficient to show that $f(s)$ satisfies the conditions of Theorem 1 (or Theorem 2), and those in Theorem 3 which deal with the character of $f(s)$ in $\cap(s) \leqq \gamma^{12}$ ). A much simpler but more restrictive set of conditions can be stated as follows:

Theorem 4. If $f(s)$ is analytic in the half-plane $R(s) \geqq \gamma$ and its singularities are ali poles, and if for some fixed $k>1$ it is true that $\left|s^{k} f(s)\right|$ is bounded in this half-plane and at all points on some sequence of circles $|s|=\varrho_{n}$ where $\varrho_{n}$ becomes infinite with $n$, then the series (5)

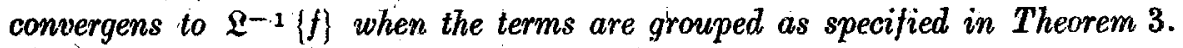

If $M$ is an upper bound of $\left|s^{k} f(s)\right|$ in $\Re(s) \geqq \gamma$ then

$$
|f(\sigma+\omega i)| \leqq M /\left(\sigma^{2}+\omega^{2}\right)^{k / 2} \leqq M /|\sigma|^{k},
$$

12) It is of interest to note that when the conditions of Theorem 1 and those of Theorem 3 with $k>1$ are satisfied, the series (5) also converges for $t=0$. 
and since $k>1$ the conditions in Theorem 2 are satisfied. All conditions in Theorem 3 are clearly satisfied so Theorem 4 is established.

By employing rectangles instead of circular segments the following useful conditions can be shown to be sufficient:

Theorem 5. Let $f(s)$ satisfy the conditions:

$1^{0} f(s)$ is analytic everwhere except in the strip $\mu<\Re(s)<\gamma$, and its singular points consist only of poles.

$2^{0}$ For some fixed $k>1,\left|s^{k} f(s)\right|$ is bounded in $\Re(s) \geqq \gamma$.

$3^{0}|f(s)|$ is bounded in $\Re(s) \leqq \mu$.

$4^{0}$ For some system of horizontal lines $\mathfrak{I}(s)= \pm \omega_{n}$, where $\omega_{n}$ becomes infinite with $n$, it is true that $\lim _{n \rightarrow \infty}\left|f\left(\sigma \pm \omega_{n} i\right)\right|=0$ uniformly for all $\sigma \leqq \gamma$

Then the series (5) converges for $t>0$ to $\mathfrak{L}^{-1}\{f(s)\}$ provided its terms which correspond 10 the poles in each pair of strips $\omega_{n}<\mathfrak{I}(s)$ $<\omega_{n+1}$ and $-\omega_{n+1}<\mathfrak{J}(s)<-\omega_{n}$ are grouped as a single term.

The integral of $e^{s t} f(s) / 2 \pi i$ in the positive direction around the contour of the rectangle with vertices $\gamma+\omega_{n} i,-\omega_{n}+\omega_{n} i,-\omega_{n}-\omega_{n} i, \gamma-\omega_{n} i$ is the sum of the residues represented by the right-hand member of (7), where $m_{n}$ is the number of poles within the rectangle. The integral along the upper side approaches zero as $n \rightarrow \infty$ since, according to $4^{0}$, for any $\varepsilon>0$ a number $N$ can be found such that

$$
\left|\int_{\gamma+\omega_{n} i}^{-\omega_{n}+\omega_{n} i} e^{s t} f(s) d s\right| \leqq \int_{-\omega_{n}}^{\gamma} e^{\sigma t}\left|f\left(\sigma+\omega_{n} i\right)\right| d \sigma \leqq \varepsilon \frac{e^{\gamma t}-e^{-\omega_{n} t}}{t}<\varepsilon e^{\gamma t} / t
$$

for $n>N$. Similarly for the integral along the lower side of the rectangle. The integral along the left hand side also approaches zero as $n \rightarrow \infty$ :

$$
\left|\int_{-\omega_{n}+\omega_{n} i}^{-\omega_{n}-\omega_{n} i} e^{s t} f(s) d s\right| \leqq e^{-\omega_{n} t} \int_{-\omega_{n}}^{\omega_{n}}\left|f\left(-\omega_{n}+\omega i\right)\right| d \omega \leqq 2 M \omega_{n} e^{-\omega_{n} t}<\varepsilon^{\prime}
$$

for $n$ sufficiently large, where $M$ is an upper bound of $|/(s)|$ in $\mathfrak{R}(s) \leqq \mu$ (condition $3^{0}$ ).

The contour integral therefore approaches

$$
\frac{1}{2 \pi i} \int_{\gamma-\omega_{n} i}^{\gamma+w_{n} i} e^{s i} f(s) d s
$$

when $n$ is large. As $n \rightarrow \infty$ this integral converges to $\mathfrak{R}^{-1}\{f(s)\}$ under the hypotheses $1^{0}$ and $2^{0}$ (this can be seen from Theorem 2), so it follows that the series (5) for $t>0$ converges to $\mathfrak{E}^{-1}\{f\}$ when the terms are grouped as stated in the theorem. 
Some of the conditions given in this theorem are more restrictive than necessary for this type of proof; they are so written to simplify the theorem and still cover an important group of functions which appear in boundary-value problems. The condition in $1^{0}$ for instance, that all poles of $f(s)$ lie to the right of a line, $\Re(s)=\mu$, can be removed if the vertical lines which form the left-hand sides of the rectangles are properly specified and if $3^{\circ}$ is stated for all points on these lines. Condition $2^{\circ}$ can also be replaced by the less restrictive conditions for the representation of $\mathfrak{L}^{-1}\{f\}$ in the integral form (4). On the other hand the three conditions $2^{0}, 3^{0}, 4^{0}$ can be replaced by this sufficient condition: that for some $k>1,\left|s^{k} f(s)\right|$ is bounded at all points in the two halfplanes $\Re(s) \geqq \gamma, \Re(s) \leqq \mu$ and on the lines $\mathfrak{\Im}(s)= \pm \omega_{n}$.

When the poles $s_{m}$ of $f(s)$ are all simple with residues $A_{m}$ the expansion formula (5) reduces to

$$
\mathfrak{E}^{-1}\{f(s)\}=\sum_{m=1}^{\infty} A_{m} e^{i_{m} t} \quad(t>0) .
$$

\section{$\S 4$.}

\section{The inverse transformations of two special functions.}

Functions involving the ratios of $\sinh \alpha s$ and $\cosh \beta s$ are prominent in the solution of the Laplace transformation of the wave equation; this will be illustrated in $\$ 5$. Simple formulas for the inverse transformations of some of these can be given with the aid of the above expansion formula.

Consider the function

$$
q(s)=\frac{\sinh \alpha s}{s^{2} \cosh \beta s}
$$

$$
(|\alpha|<\beta),
$$

where $\alpha$ und $\beta$ are real and $\beta$ can be considered positive. The singular points are the simple poles $s=0$ and $s=s_{m}=\lambda_{m} i / \beta$, where

$$
\lambda_{m}=(2 m-1) \pi / 2, \quad m=0, \pm 1, \pm 2, \ldots
$$

Hence $q(s)$ is analytic in the half-plane $\Re(s) \geqq \gamma$ where $\gamma$ is any positive constant, (and also in $\mathfrak{R}(s) \leqq \mu$ where $\mu<0$ ). In $\mathfrak{R}(s) \geqq \gamma,\left|s^{2} q(s)\right|$ is bounded since

$$
\left|s^{2} q(s)\right|=\left|\frac{e^{-(\beta-\alpha) s}-e^{-(\beta+\alpha) s}}{1+e^{-2 \beta s}}\right|<\frac{2}{1-e^{-2 \beta \gamma}} .
$$

The sequence of circles of Theorem 4 can be taken as $|s|=n \pi / \beta$, $(n=1,2, \ldots)$. When the value of $\left|s^{2} q(s)\right|^{2}$ at points on these circles is written by putting $s=n \pi e^{i \theta} / \beta$, it can be shown by elementary processes that this function is bounded for any method of variation of the independent variables $n$ and $\theta, q(s)$ therefore satisfies the conditions in Theorem 4. 
It is easier in this case to show that the function satisfies the conditions in Theorem 5 . In $\Re(s) \leqq \mu<0$,

$$
\left|s^{2} q(s)\right|=\left|\frac{e^{(\alpha+\beta) s}-e^{(-\alpha+\beta) \delta}}{e^{2 \beta \gamma}+1}\right|<\frac{2}{1-e^{2 \beta \mu}}
$$

so the condition $3^{0}$ is satistied. On the lines $\mathfrak{I}(s)= \pm n \pi / \beta, s=\sigma \pm i n \pi / \beta$ and when $\sigma \leqq \gamma$,

$$
|q(s)| \leqq \frac{e^{\alpha \sigma}+e^{-\alpha \sigma}}{|s|^{2}\left(e^{\beta \sigma}+e^{-\beta \sigma}\right)}<\frac{e^{(\beta+\alpha) \gamma}+e^{(\beta-\alpha) \gamma}}{\omega_{n}^{2}}
$$

so that $4^{\circ}$ is satisfied. It was shown above that the other conditions in Theorem 5 are fulfilled by $q(s)$.

According to the expansion (8) then

$$
\begin{aligned}
Q^{-1}\{q(s)\} & =\alpha+\sum_{m=-\infty}^{\infty} \frac{\sinh \left(\alpha s_{m}\right) e_{m}^{z}}{\beta s_{m}^{y} \sinh \left(\beta s_{m}\right)} \\
& =\alpha+2 \beta \sum_{m=1}^{\infty}(-1)^{m} \sin \left(\lambda_{m} \alpha / \beta\right) \cos \left(\lambda_{m} t / \beta\right) / \lambda_{m}^{2},
\end{aligned}
$$

where $\lambda_{m}=(2 m-1) \pi / 2$. The terms corresponding to poles with opposite signs have been grouped according to Theorem 3 (and also Theorem 5). This series can be written in the form

$$
\mathfrak{R}^{-1}\{q(s)\}=\alpha+\beta \sum_{m=1}^{\infty}(-1)^{m}\left[\sin \frac{\lambda_{m}(\alpha-i)}{\beta}+\sin \frac{\lambda_{m}(\alpha+i)}{\beta}\right] / \lambda_{m}^{2},
$$

which can be recognized as the sum of two simple Fourier series. When $\alpha=x / c$ and $\beta=l / c(l>0, c>0)$ formula (10) can be written

$$
\left.\mathcal{Q}-1 \mid \frac{\sinh (s x / c)}{\left.s^{2} \cosh s l / c\right)}\right\}=\frac{x}{c}-\frac{1}{2 c}[G(x-c t)+G(x+c t)], \quad|x|<z
$$

where $G(u)$ is the broken line function defined by the series

or by the conditions

$$
G(u)=\frac{8 l}{\pi^{2}} \sum_{n=1,3,5, \ldots}^{\infty} \frac{1}{n^{2}} \sin \frac{n \pi}{2} \sin \frac{n \pi u}{2 l}
$$

$$
G(u)=-G(-u)=G(u+4 l), \quad G(u)=\left\{\begin{array}{l}
u \text { if } 0 \leqq u \leqq l, \\
2 l-u \text { if } l \leqq u \leqq 2 l
\end{array}\right.
$$

According to the differentiation property (2),

$$
s q(s)-Q(0)=\mathfrak{Q}\left\{Q^{\prime}(t)\right\}
$$

where $Q(t)$ is the right-hand member of $(11)$. Since $Q(0)=0$ it follows that

$$
Q^{-1}\{s q(s)\}=Q^{\prime}(t)
$$

and the second special inversion of importance can be written

$$
\mathfrak{I}^{-1}\left\{\frac{\sinh (s x i c)}{s \cosh (s l / c)}\right\}=\frac{1}{2}[H(x-c t)-H(x-c t)] \quad|x|<l,
$$


where the broken line function $H(u)=G^{\prime}(u)$ is defined by the conditions

$$
H(u)=H(-u)=H(u+4 l), \quad H(u)=\left\{\begin{aligned}
1 & \text { if } 0<u<l, \\
-1 & \text { if } l<u<2 l .
\end{aligned}\right.
$$

The inversion (14) can be verified by applying $\mathfrak{L}$ to both members and performing the integration.

The expansion (5) gives a similar inversion formula for the function

$$
\frac{\cosh (s x / c)}{s^{2} \sinh (s / / c)}
$$

which has a pole of the third order at $s=0$. This function arises in the problem of forced displacements in a stretched string.

$\S 5$.

\section{A problem of displacements in a bar.}

The Laplace transformation and the results of the last section can be used to find a simple formula for the longitudinal displacements in a prismatic bar with one end fixed and a variable force, acting on the other end. Let the origin be taken in the fixed end and let $Y(x, t)$ be the displacement along the bar at time $t$ of a point originally at a distance $x$ from this end. If $l$ denotes the length of the bar and $c^{2}$ the usual elastic coefficient, then

$$
\begin{array}{rlrl}
Y_{t t}=c^{2} Y_{x x} & (0<x<l), \\
\lim _{t \rightarrow 0} Y(x, t)=\lim _{t \rightarrow 0} Y_{t}(x, t) & =0, & \\
\lim _{x \rightarrow 0} Y(x, t)=0, & \lim _{x \rightarrow l} Y_{x}(x, t)=F(t),
\end{array}
$$

where $F(t)$ is proportional to the force acting on the end $x=l$.

Let $y(x, s)$ be the Laplace transform, with respect to $t$, of $Y(x, t)$. The application of 2 to (16) and (18) gives, in view of $(2)$ and (17), these corresponding conditions $\left.{ }^{13}\right)$ on $y(x, s)$ :

$$
\begin{gathered}
s^{2} y(x, s)=c^{2} y_{x x}(x, s), \\
\lim _{x \rightarrow 0} y(x, s)=0, \quad \lim _{x \rightarrow l} y_{x}(x, s)=f(s),
\end{gathered}
$$

where $f(s)=\mathfrak{L}\{F(t)\}$. This is a boundary-value problem in ordinary differential equations with the parameter $s$. Its solution is

$$
y(x, s)=\operatorname{cf}(s) \frac{\sinh (s x / c)}{s \cosh (s l / c)},
$$

13) According to these steps we aro seeking a solution whose second derivative in $t$ has a Laplace transformation and for which the derivatives and limits with respect to $x$ involved here are commutable with respect to the transformation $\mathfrak{E}$. See the papers cited under ${ }^{3}$ ) for a further examination of such steps. 
so. the displacement is

$$
Y(x, t)=c \mathfrak{Q}^{-1}\left\{f(s) \frac{\sinh (s x / c)}{s \cosh \left(s l / e^{\prime}\right)}\right\} .
$$

The inverse transformation of the product of two functions is given in the following way by the composition (Faltung) integral ${ }^{14}$ ): if

then

$$
\mathfrak{L}^{-1}\left\{f_{1}(s)\right\}=F_{1}(t) \text { and } \mathfrak{L}^{-x}\left\{f_{2}(s)\right\}=F_{2}(t)
$$

$$
\mathcal{L}-\left\{f_{1}(s) f_{2}(s)\right\}=\int_{0}^{t} F_{1}(\tau) F_{2}(t-\tau) d \tau=\int_{0}^{t} F_{1}(t-\tau) F_{2}(\tau) d \tau .
$$

By applying this to the inversion in (19) and noting that $\left.\mathfrak{2}^{-1} \mid f(s)\right\}=F(l)$ while the inversion of the second factor is given by (14), the displacement is found to be

$$
Y(x, t)=\frac{c}{2} \int_{0}^{t} F(\tau)[H(x-c t+c \tau)-H(x+c t-c \tau)] d \tau
$$

where the function $H(u)$ is defined by the conditions (15). This can be written

$$
Y(x, t)=\frac{1}{2} \int_{x-c t}^{x} F\left(\frac{c t+u-x}{c}\right) H(u) d u-\frac{1}{2} \int_{x}^{x+c t} F\left(\frac{c t-u+x}{c}\right) H(u) d u,
$$

and in this form the integrals can be expanded to show that the conditions (16) to (18) are satisfied ${ }^{25}$ ).

It is of interest to note that if the force is constant, $F(t)=K$, the displacèment formula becomes

$$
Y(x, t)=K x-\frac{K}{2}[G(x-c t)+G(x+c t)]
$$

It follows from the description (13) of $G(u)$ that $G(l-c t)=G(l+c t)$, and hence at the end $x==l$

$$
Y(l, t)=K[l-G(l+c t)]
$$

which is the simple vibration expected.

14) Conditions on $F_{1}$ and $F_{2}$ under which the composition theorem is valid are given in the second paper cited under ${ }^{1}$ ). Conditions on $f_{1}$ and $f_{2}$ for the validity of the above form of the theorem are given by W. v. Koppenfels, Der Faltungssatz und seine Anwendung bei der Integration linearer Differentialgleichungen mit konstanten Koeffizienten. Math. Annalen 105 (1931), 694-706.

$\left.{ }^{15}\right)$ The inversion process used by Doetsch in the second paper cited under ${ }^{3}$ ) can also be used here; it expresses the displacement by means of a finite series in which the number of terms depends on the values of $x$ and $t$. 